ISSN 2075-9827 e-ISSN 2313-0210

Carpathian Math. Publ. 2020, 12 (1), 208-228

doi:10.15330/cmp.12.1.208-228 http://www.journals.pnu.edu.ua/index.php/cmp

Карпатські матем. публ. 2020, Т.12, №1, С.208-228

SOLTANOV K. ${ }^{1}$, SERT U. ${ }^{2}$

\title{
CERTAIN RESULTS FOR A CLASS OF NONLINEAR FUNCTIONAL SPACES
}

\begin{abstract}
In this article, we study properties of a class of functional spaces, so-called pn-spaces, which arise from investigation of nonlinear differential equations. We establish some integral inequalities to analyse the structures of the pn-spaces with the constant and variable exponent. We prove embedding theorems, which indicate the relation of these spaces with the well known classical Lebesgue and Sobolev spaces with the constant and variable exponents.

Key words and phrases: pn-space, variable exponent, integral inequality, nonlinear differential equation, embedding theorem.
\end{abstract}

${ }^{1}$ Department of Mathematics, Iğdır University, 76000, Iğdır, Turkey

${ }^{2}$ Department of Mathematics, Hacettepe University, 06800, Beytepe, Ankara, Turkey

E-mail: kamal.soltanov@igdir.edu.tr (Soltanov K.), usert@hacettepe.edu.tr (Sert U.)

\section{INTRODUCTION}

This paper is concerned with some features of a class of functional spaces 6 which are emerged from investigation of nonlinear differential equations. Studying boundary value problems (BVPs) require to examine and understand the functional spaces 6 which are directly related with the considered problem. In other words, it is required to work on the domain of the operator generated by the addressed boundary value problem. We specify that it is better to study each BVPs on its own space. Furthermore, detailed analysis of these spaces and examining their topology, structure etc. cause to gain better results of the possed problem (for example, regularity of the solution).

The spaces generated by boundary value problems for the linear differential equations are generally linear spaces such as Sobolev spaces and different generalizations of them. Apart from boundary value problems for linear differential equations, the spaces generated by nonlinear differential equations (essentially the domain of the corresponding operator) are subsets of linear spaces and do not have linear structure. The class of spaces of this type were introduced and investigated by Soltanov in the abstract case (see, e.g. [21-26]), and also in the case of functions spaces (see, e.g. [23-30] and references therein, where various subsets of linear spaces of this type were searched). In the mentioned articles, topology of these spaces were investigated and shown that under what circumstances they are metric or pseudo-metric spaces. Starting from these features of the introduced spaces, they were defined as the class of pseudonormed spaces or pn-spaces and the class of quasi-pseudo normed spaces or qn-spaces.

In this work, we focus on the characteristics of certain class of functional pn-spaces. Essentially, we deal with the following class of functional pn-spaces.

2010 Mathematics Subject Classification: Primary 46A99, 46E30, 46E35, 46T99; Secondary 26D20, 26D99, 35D30, 35J62, 35K61. 
Let $\Omega \subset \mathbb{R}^{n}(n \geq 1)$ be bounded domain with sufficiently smooth boundary. In this work the class of functions $u: \Omega \longrightarrow \mathbb{R}$ of the following type will be investigated

$$
S_{m, \alpha, \beta}(\Omega):=\left\{u \in L^{1}(\Omega):[u]_{S_{m, \alpha, \beta}(\Omega)}^{\alpha+\beta}<\infty\right\},
$$

where

$$
[u]_{S_{m, \alpha, \beta}(\Omega)}^{\alpha+\beta}:=\sum_{0 \leq|k| \leq m}\left(\int_{\Omega}|u|^{\alpha}\left|D^{k} u\right|^{\beta} d x\right), \quad D=\left(D_{1}, D_{2}, \ldots, D_{n}\right),
$$

$D_{i}=\frac{\partial}{\partial x_{i}}, D^{k} \equiv D_{1}^{k_{1}} D_{2}^{k_{2}}, \ldots, D_{n}^{k_{n}}, i=\overline{1, n},|k|=\sum_{i=1}^{n} k_{i}$. Here, we only address the cases $m=1,2$.

It is important to note that the following subset of $L^{p}(\Omega), p \geq 2$,

$$
M:=\left\{u \in L^{1}(\Omega): \sum_{i=1}^{n}\left(\int_{\Omega}|u|^{p-2}\left|D_{i} u\right|^{2} d x\right)<\infty, u \mid \partial \Omega=0\right\}
$$

was arose in the article of Dubinskii earlier $([7,8,11])$ while studying the following nonlinear problem

$$
\begin{gathered}
\frac{\partial u}{\partial t}-\sum_{i=1}^{n} D_{i}\left(|u|^{p-2} D_{i} u\right)=h(x, t), \quad(t, x) \in(0, T) \times \Omega, \\
u(0, x)=u_{0}(x),\left.\quad u\right|_{(0, T] \times \partial \Omega}=0 .
\end{gathered}
$$

Here, compact inclusion of subset $M$ to the space $L^{p}(\Omega)$ and also necessary compactness theorems for analysis of the parabolic problem were proved. Later on, different new subsets of $L^{1}(\Omega)$ appeared in the articles of Soltanov (see, e.g. [23-25]) while studying the mixed problem for the following nonlinear equation, which is type of the Prandtl-von-Mises equation

$$
\frac{\partial u}{\partial t}-|u|^{\rho} \frac{\partial^{2} u}{\partial x^{2}}=h(t, x), \rho>0, \quad(t, x) \in(0, T) \times \Omega .
$$

For example, one of the emerged class in the case of $\Omega=(a, b) \subset \mathbb{R}$ can be expressed in the form

$$
\left\{u \in L^{1}(\Omega): \int_{\Omega}|u|^{\alpha}\left|D^{2} u\right|^{\beta} d x<\infty, u(a)=u(b)=0\right\},
$$

and also as type of subsets in the form $S_{m, \alpha, \beta}(\Omega)$. Here, we specify that different problems to the equation (1) were studied under various additional conditions as well (see, e.g. $[12,14,18$, 35-37]).

Accordingly, in the papers [24,25] etc. different classes of sets of this type were examined and it was shown that these sets are nonlinear topological spaces, moreover they are either metric or pseudo-metric spaces. Many other properties of the introduced spaces were investigated as well in these works. For instance, relations of these spaces amongst themselves and with well known functional spaces (e.g. Lebesgue or Sobolev spaces etc).

Consequently, in the mentioned works pn-spaces and qn-spaces were defined with taking into account the principal attributes of the presented spaces.

These spaces may arise from the research of the existence of smooth solution of the following differential equation

$$
-\Delta u+u+|u|^{p} u=h(x), x \in \Omega \subset \mathbb{R}^{n}, n \geq 2,
$$




$$
\left.\left(\frac{\partial u}{\partial \eta}+|u|^{\mu} u\right)\right|_{\partial \Omega}=\psi\left(x^{\prime}\right), x^{\prime} \in \partial \Omega, p, \mu \geq 0,
$$

which was studied by Soltanov [32]. We emphasize that equation of this form was considered by many authors, who tried to answer various questions of different problems for this equation, (see, e.g. Berestycki ve Nirenberg [3], Brezis [4], etc.). In [15], Pohozaev employed another approach for this problem that led to gaining distinct results other than [32].

This kind of nonlinear spaces are generated by the differential equations, which ensue from the mathematical models of some processes in flood mechanics. For example, we may present the nonlinear equation of type

$$
\frac{\partial u}{\partial t}-|u|^{p-2} \Delta u=h(x, t), p \geq 2,
$$

where this equation were studied [24,31] and [33]. Similar equations were handled by Oleynik [14], Walter [36] only using the approximation way and Tsutsumi, Ishiwata [35] focused on understanding the behavior of the solution.

In recent years, there have been an increasing interest in the study of equations with variable exponents of nonlinearities. The interest in the study of differential equations that involves variable exponents is motivated by their applications to the theory of elasticity and hydrodynamics, in particular, the models of electrorheological fluids [17] in which a substantial part of viscous energy, the thermistor problem [38], image processing [5] and modeling of non-Newtonian fluids with thermo-convective effects [2] etc.

In the most of these papers, that concern with equations, which have non standard growth, authors studied the problems, which involve $p($.$) -Laplacian type equation or equations, which$ fulfill monotonicity conditions, where enable to apply monotonicity methods. Unlike these works, in the articles $[19,20]$ investigating some properties of nonlinear spaces with variable exponent, we developed an approach based on the spaces corresponding to problem under consideration. It is necessary to note, that the questions mentioned above may arise for the problems, which have variable exponent nonlinearity. Eventually, here we also study variable exponent nonlinear spaces that are essential for the investigation of the following type of equations

$$
\nabla \cdot\left[\left(|\nabla u|^{p_{0}(x)-2}+|u|^{p_{1}(x)-2}\right) \nabla u\right]=h(x, u) .
$$

Since we want to establish the regularity of solution of the nonlinear differential equations related with mentioned pn-spaces, thus our aim is to understand the structure and nature of these spaces better, that allows to investigate the characteristics of solutions. For this reason, in this article we prove some embedding results, which indicate the relation of these spaces between Sobolev and Lebesgue spaces. We show that these spaces are not merely subsets of Lebesgue spaces also subsets of Sobolev spaces.

This paper is organized as follows. In the next section, we give the definitions of certain type of pn-spaces with variable and constant exponents $([20,33]$ and for general definition see [34]) as well as recall some basic results for these spaces and variable exponent spaces. In Section 2, we prove embedding theorems for constant exponent pn-spaces and give certain results with examples in one dimensional case. In Section 3 firstly, we establish some integral inequalities with variable exponents, which are required to prove embedding theorems of variable exponent nonlinear spaces then investigate some attributes of variable exponent pn-spaces. 


\section{PRELIMINARIES}

In this section in the beginning we will give the general definition of spaces that are studied here in the functional case. Let $X, Y$ be locally convex vector topological spaces, $B \subseteq Y$ be a Banach space and $g: D(g) \subseteq X \longrightarrow Y$. Let's introduce the following subset of $X$

$$
\mathcal{M}_{g B} \equiv\{x \in X: g(x) \in B, \operatorname{Im} g \cap B \neq \varnothing\} .
$$

Definition 1. A subset $\mathcal{M} \subseteq X$ is called a pn-space (i.e. pseudonormed space) if $S$ is a topological space and there is a function $[\cdot]_{\mathcal{M}}: \mathcal{M} \longrightarrow \mathbb{R}_{+}^{1} \equiv[0, \infty)$ (which is called $p$-norm of $\mathcal{M}$ ) such that

qn) $[x]_{\mathcal{M}} \geq 0, \forall x \in \mathcal{M}$ and $x=0 \Longrightarrow[x]_{\mathcal{M}}=0$;

pn) $\left[x_{1}\right]_{\mathcal{M}} \neq\left[x_{2}\right]_{\mathcal{M}} \Longrightarrow x_{1} \neq x_{2}$ for $x_{1}, x_{2} \in \mathcal{M}$, and $[x]_{\mathcal{M}}=0 \Longrightarrow x=0$.

The following conditions are often fulfilled in the spaces $\mathcal{M}_{g B}$.

$\mathrm{N}_{1}$ ) There exist a convex function $v: \mathbb{R}^{1} \longrightarrow \overline{\mathbb{R}_{+}^{1}}$ and number $K \in(0, \infty]$ such that $[\lambda x]_{\mathcal{M}} \leq$ $v(\lambda)[x]_{\mathcal{M}}$ for any $x \in \mathcal{M}$ and $\lambda \in \mathbb{R}^{1},|\lambda|<K$, moreover, $\lim _{|\lambda| \longrightarrow \lambda_{j}} \frac{v(\lambda)}{|\lambda|}=c_{j}, j=0,1$, where $\lambda_{0}=0, \lambda_{1}=K$ and $c_{0}=c_{1}=1$ or $c_{0}=0, c_{1}=\infty$, i.e. if $K=\infty$ then $\lambda x \in \mathcal{M}$ for any $x \in \mathcal{M}$ and $\lambda \in \mathbb{R}^{1}$.

Let $g: D(g) \subseteq X \longrightarrow Y$ be such a mapping that $\mathcal{M}_{g B} \neq \varnothing$ and the following conditions are fulfilled

$\left.\mathrm{G}_{1}\right) g: D(g) \longleftrightarrow \operatorname{Im} g$ is a bijection and $g(0)=0$;

$\mathrm{G}_{2}$ ) there is a function $v: \mathbb{R}^{1} \longrightarrow \overline{\mathbb{R}_{+}^{1}}$ satsfying the condition $\mathrm{N}_{1}$ such that

$$
\|g(\lambda x)\|_{B} \leq v(\lambda)\|g(x)\|_{B}, \forall x \in \mathcal{M}_{g B}, \forall \lambda \in R^{1}
$$

If the mapping $g$ satisfies the conditions $G_{1}$ and $G_{2}$ then $\mathcal{M}_{g B}$ is a pn-space with $p$-norm defined in the following way: there is a one-to-one function $\psi: \mathbb{R}_{+}^{1} \longrightarrow \mathbb{R}_{+}^{1}, \psi(0)=0$, $\psi, \psi^{-1} \in C^{0}$ such that $[x]_{\mathcal{M}_{g B}} \equiv \psi^{-1}\left(\|g(x)\|_{B}\right)$. In this case $\mathcal{M}_{g B}$ is a metric space with a metric: $d_{\mathcal{M}}\left(x_{1} ; x_{2}\right) \equiv\left\|g\left(x_{1}\right)-g\left(x_{2}\right)\right\|_{B}$. Further, we consider just such type of pn-spaces.

Definition 2. The pn-space $\mathcal{M}_{g B}$ is called weakly complete if $g\left(\mathcal{M}_{g B}\right)$ is weakly closed in $B$. The pn-space $\mathcal{M}_{g B}$ is "reflexive" if each bounded weakly closed subset of $\mathcal{M}_{g B}$ is weakly compact in $\mathcal{M}_{g B}$.

It is clear that if $B$ is a reflexive Banach space and $\mathcal{M}_{g B}$ is a weakly complete pn-space, then $\mathcal{M}_{g B}$ is "reflexive". Moreover, if $B$ is a separable Banach space, then $\mathcal{M}_{g B}$ is separable, also. For complementary properties see, e.g. [23,33,34].

We now remind certain integral inequalities and facts about the functional pn-spaces with constant exponent that are concerned in this paper (for general case see [21-25] and for functional case $[21,25,27]$ etc).

Let $\Omega \subset \mathbb{R}^{n}(n \geq 1)$ be a bounded domain with Lipschitz boundary $\partial \Omega$. Throughout the paper, we denote by $|\Omega|$ the Lebesgue measure of $\Omega$. 
Lemma 1. Let $\alpha \geq 0, \beta \geq 1,|\Omega|<\infty$ and $i=\overline{1, n}$, then for all $u \in C(\bar{\Omega}) \cap C^{1}(\Omega)$ the inequality

$$
\int_{\Omega}|u|^{\alpha+\beta} d x \leq C_{1} \int_{\Omega}|u|^{\alpha}\left|D_{i} u\right|^{\beta} d x+C_{2} \int_{\partial \Omega}|u|^{\alpha+\beta} d x^{\prime}
$$

is satisfied. Here, $C_{1}=C_{1}(\alpha, \beta,|\Omega|), C_{2}=C_{2}(|\Omega|)>0$ are constants.

Lemma 2. Assume that $\alpha, \alpha_{1} \geq 0, \beta \geq 1$ and $\beta>\beta_{1}>0, \frac{\alpha_{1}}{\beta_{1}} \geq \frac{\alpha}{\beta}, \alpha_{1}+\beta_{1} \leq \alpha+\beta$ be satisfied. Then for $u \in C(\bar{\Omega}) \cap C^{1}(\Omega)$

$$
\int_{\Omega}|u|^{\alpha_{1}}\left|D_{i} u\right|^{\beta_{1}} d x \leq C_{3} \int_{\Omega}|u|^{\alpha}\left|D_{i} u\right|^{\beta} d x+C_{4} \int_{\partial \Omega}|u|^{\alpha+\beta} d x^{\prime}+C_{5}
$$

holds. Here, for $r=3,4,5, C_{r}=C_{r}\left(\alpha, \beta, \alpha_{1}, \beta_{1},|\Omega|\right)>0$ are constants.

Lemma 3. Let $\alpha \geq 0, \beta_{0}+\beta_{1} \geq 2$ and $\beta_{1} \geq \beta_{0} \geq 0$ be fulfilled. Then for all $u \in C^{1}(\bar{\Omega}) \cap C^{2}(\Omega)$

$$
\begin{aligned}
\int_{\Omega}|u|^{\alpha}\left|D_{i} u\right|^{\beta_{0}+\beta_{1}} d x & \leq C_{6} \int_{\Omega}|u|^{\alpha+\beta_{0}}\left|D_{i}^{2} u\right|^{\beta_{1}} d x \\
& +C_{7} \int_{\partial \Omega}\left(|u|^{\alpha+\beta_{0}+\beta_{1}}+|u|^{\alpha+1}\left|D_{i} u\right|^{\beta_{0}+\beta_{1}-1}\right) d x^{\prime}
\end{aligned}
$$

holds. Here, for $j=6,7, C_{j}=C_{j}\left(\alpha, \beta, \beta_{0}\right)>0$ are constants.

Definition 3. Let $\alpha \geq 0, \beta \geq 1, \mathbf{k}=\left(k_{1}, \ldots, k_{n}\right)$ be multi-index and $|\mathbf{k}|=\sum_{i=1}^{n} k_{i}, m \in \mathbb{Z}^{+}$, $\Omega \subset \mathbb{R}^{n}(n \geq 1)$ is bounded domain with sufficiently smooth boundary (at least Lipschitz boundary)

$$
S_{m, \alpha, \beta}(\Omega):=\left\{u \in L^{1}(\Omega):[u]_{S_{m, \alpha}, \beta}^{\alpha+\beta} \equiv \sum_{0 \leq|\mathbf{k}| \leq m}\left(\int_{\Omega}|u|^{\alpha}\left|D^{\mathbf{k}} u\right|^{\beta} d x\right)<\infty\right\}
$$

and

$$
\stackrel{\circ}{S}_{m, \alpha, \beta}(\Omega):=S_{m, \alpha, \beta}(\Omega) \cap\left\{D^{\mathbf{k}_{u}}|\partial \Omega \equiv 0,0 \leq| \mathbf{k} \mid \leq m_{0}<m\right\} .
$$

We state a proposition which can be easily proved by the help of Lemmas 1-3 and Definition 3.

Proposition 1. Assume that $\alpha \geq 0, \beta \geq 1$, then we have the following equivalence

$$
\stackrel{\circ}{S}_{1, \alpha, \beta}(\Omega):=\left\{u \in L^{1}(\Omega):[u]_{S_{1, \alpha, \beta}(\Omega)}^{\alpha+\beta} \equiv \sum_{i=1}^{n}\left(\int_{\Omega}|u|^{\alpha}\left|D_{i} u\right|^{\beta} d x\right)<\infty\right\}
$$

and $^{1}$

$$
\stackrel{\circ}{S}_{2, \alpha, \beta}(\Omega):=\left\{u \in L^{1}(\Omega):[u]_{S_{2, \alpha, \beta}(\Omega)}^{\alpha+\beta} \equiv \sum_{i=1}^{n}\left(\int_{\Omega}|u|^{\alpha}\left|D_{i}^{2} u\right|^{\beta} d x\right)<\infty\right\} .
$$

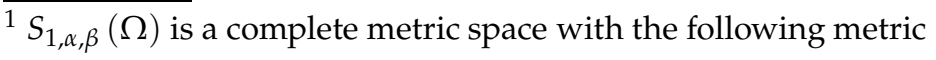

$$
d_{S_{1, \alpha, \beta}}(u, v)=\left\||u|^{\frac{\alpha}{\beta}} u-|v|^{\frac{\alpha}{\beta}} v\right\|_{W^{1, \beta}(\Omega)}, \quad \forall u, v \in S_{1, \alpha, \beta}(\Omega) .
$$


Theorem 1. Let $\alpha \geq 0, \beta \geq 1$, then $g: \mathbb{R} \longrightarrow \mathbb{R}, g(t):=|t|^{\frac{\alpha}{\beta}} t$ is an one to one correspondence from $S_{1, \alpha, \beta}(\Omega)$ onto $W^{1, \beta}(\Omega)$.

Now, we recall some basic definitions and results about variable exponent Lebesgue and Sobolev spaces $[1,6,9,10,13]$.

Let $\Omega$ be a Lebesgue measurable subset of $\mathbb{R}^{n}$ such that $|\Omega|>0$. The function set $M(\Omega)$ denotes the family of all measurable functions $p: \Omega \longrightarrow[1, \infty]$ and the set $M_{0}(\Omega)$ is defined by

$$
M_{0}(\Omega):=\left\{p \in M(\Omega): 1 \leq p^{-} \leq p(x) \leq p^{+}<\infty \text {, a.e. } x \in \Omega\right\},
$$

where $p^{-}:=\underset{\Omega}{\operatorname{ess} \inf }|p(x)|, p^{+}:=\underset{\Omega}{\operatorname{ess} \sup }|p(x)|$.

For $p \in M(\Omega), \Omega_{\infty}^{p} \equiv \Omega_{\infty} \equiv\{x \in \Omega \mid p(x)=\infty\}$. On the set of all functions on $\Omega$, define the functional $\sigma_{p}$ and $\|\cdot\|_{p}$ by

$$
\sigma_{p}(u) \equiv \int_{\Omega \backslash \Omega_{\infty}}|u|^{p(x)} d x+\underset{\Omega_{\infty}}{\operatorname{ess} \sup }|u(x)|
$$

and

$$
\|u\|_{L^{p(x)}(\Omega)} \equiv \inf \left\{\lambda>0: \sigma_{p}\left(\frac{u}{\lambda}\right) \leq 1\right\} .
$$

If $p \in L^{\infty}(\Omega)$, then $p \in M_{0}(\Omega), \sigma_{p}(u) \equiv \int_{\Omega}|u|^{p(x)} d x$ and the variable exponent Lebesgue space is defined as follows

$$
L^{p(x)}(\Omega):=\left\{u: u \text { is a measurable real-valued function such that } \sigma_{p}(u)<\infty\right\} .
$$

If $p^{-}>1$, then the space $L^{p(x)}(\Omega)$ becomes a reflexive and separable Banach space with the norm $\|\cdot\|_{L^{p(x)}(\Omega)}$, which is so-called Luxemburg norm.

If $0<|\Omega|<\infty$, and $p_{1}, p_{2} \in M(\Omega)$, then the continuous embedding $L^{p_{1}(x)}(\Omega) \subset$ $L^{p_{2}(x)}(\Omega)$ exists $\Longleftrightarrow p_{2}(x) \leq p_{1}(x)$ for a.e. $x \in \Omega$.

For $u \in L^{p(x)}(\Omega)$ and $v \in L^{q(x)}(\Omega)$, where $p, q \in M_{0}(\Omega)$ and $\frac{1}{p(x)}+\frac{1}{q(x)}=1$, the following inequalities be satisfied

$$
\int_{\Omega}|u v| d x \leq 2\|u\|_{L^{p(x)}(\Omega)}\|v\|_{L^{q(x)}(\Omega),}
$$

and

$$
\min \left\{\|u\|_{L^{p(x)}(\Omega)}^{p^{-}},\|u\|_{L^{p(x)}(\Omega)}^{p^{+}}\right\} \leq \sigma_{p}(u) \leq \max \left\{\|u\|_{L^{p(x)}(\Omega)}^{p^{-}},\|u\|_{L^{p(x)}(\Omega)}^{p^{+}}\right\} .
$$

Lemma 4. Let $u, u_{k} \in L^{p(x)}(\Omega), k=1,2, \ldots$ Then the following statements are equivalent to each other:

1. $\lim _{k \rightarrow \infty}\left\|u_{k}-u\right\|_{L^{p(x)}(\Omega)}=0$;

2. $\lim _{k \rightarrow \infty} \sigma_{p}\left(u_{k}-u\right)=0$;

3. $u_{k}$ converges to $u$ in $\Omega$ in measure and $\lim _{k \rightarrow \infty} \sigma_{p}\left(u_{k}\right)=\sigma_{p}(u)$. 
Let $\Omega \subset \mathbb{R}^{n}$ be a bounded domain and $p \in L^{\infty}(\Omega)$, then variable exponent Sobolev space is defined by

$$
W^{1, p(x)}(\Omega):=\left\{u \in L^{p(x)}(\Omega):|\nabla u| \in L^{p(x)}(\Omega)\right\}
$$

and this space is a separable Banach space with the norm

$$
\|u\|_{W^{1, p(x)}(\Omega)} \equiv\|u\|_{L^{p(x)}(\Omega)}+\|\nabla u\|_{L^{p(x)}(\Omega)} \cdot
$$

In the following discussion, we give the definition of generalized nonlinear spaces (functional pn-spaces with variable exponent) and features of them that indicate their relation with known spaces. These classes are nonlinear spaces, which are generalization of nonlinear spaces with constant exponent studied in [24] (see also references therein). We also specify that some of the results and its proofs can be found in $[19,20]$.

Definition 4. Let $\Omega \subset \mathbb{R}^{n}(n \geq 2)$ be a bounded domain with Lipschitz boundary and $\gamma, \beta$ $\in M_{0}(\Omega)$. We introduce $S_{1, \gamma(x), \beta(x)}(\Omega)$, the class of functions $u: \Omega \rightarrow \mathbb{R}$, and the functional $[\cdot]_{S_{\gamma, \beta}}: S_{1, \gamma(x), \beta(x)}(\Omega) \longrightarrow \mathbb{R}_{+}$as follows

$$
\begin{gathered}
S_{1, \gamma(x), \beta(x)}(\Omega):=\left\{u \in L^{1}(\Omega): \int_{\Omega}|u|^{\gamma(x)+\beta(x)} d x+\sum_{i=1}^{n} \int_{\Omega}|u|^{\gamma(x)}\left|D_{i} u\right|^{\beta(x)} d x<\infty\right\}, \\
{[u]_{S_{\gamma, \beta}}:=\inf \left\{\lambda>0: \int_{\Omega}\left|\frac{u}{\lambda}\right|^{\gamma(x)+\beta(x)} d x+\sum_{i=1}^{n}\left(\int_{\Omega}\left|\frac{|u|^{\frac{\gamma(x)}{\beta(x)}} D_{i} u}{\lambda^{\frac{\gamma(x)}{\beta(x)}}+1}\right|^{\beta(x)}\right) d x \leq 1\right\} .}
\end{gathered}
$$

$[\cdot]_{S_{\gamma, \beta}}$ defines a pseudo-norm on $S_{1, \gamma(x), \beta(x)}(\Omega)$, actually it can be readily verified that $[\cdot]_{S_{\gamma, \beta}}$ fulfills all axioms of pseudo-norm (see $\left.[33,34]\right)$, i.e. $[u]_{S_{\gamma, \beta}} \geq 0, u=0 \Rightarrow[u]_{S_{\gamma, \beta}}=0$, $[u]_{S_{\gamma, \beta}} \neq[v]_{S_{\gamma, \beta}} \Rightarrow u \neq v$ and $[u]_{S_{\gamma, \beta}}=0 \Rightarrow u=0$.

Let $S_{1, \gamma(x), \beta(x)}(\Omega)$ be the space given in the Definition 4 and $\theta(x) \in M_{0}(\Omega)$, we denote $S_{1, \gamma(x), \beta(x), \theta(x)}(\Omega)$, the class of functions $u: \Omega \rightarrow \mathbb{R}$, by the following intersection

$$
S_{1, \gamma(x), \beta(x), \theta(x)}(\Omega):=S_{1, \gamma(x), \beta(x)}(\Omega) \cap L^{\theta(x)}(\Omega)
$$

with the pseudo-norm

$$
[u]_{S_{\gamma, \beta, \theta}}:=[u]_{S_{\gamma, \beta}}+\|u\|_{L^{\theta(x)}(\Omega)}, \quad \forall u \in S_{1, \gamma(x), \beta(x), \theta(x)}(\Omega) .
$$

Proposition 2. If $\gamma, \beta, \theta \in M_{0}(\Omega)$ and $\theta(x) \geq \gamma(x)+\beta(x)+\varepsilon_{0}$ a.e. $x \in \Omega$ for some $\varepsilon_{0}>0$, then we have the following equivalence

$$
S_{1, \gamma(x), \beta(x), \theta(x)}(\Omega) \equiv\left\{u \in L^{1}(\Omega): R^{\gamma, \beta, \theta}(u)<\infty\right\}
$$

where $R^{\gamma, \beta, \theta}(u):=\int_{\Omega}|u|^{\theta(x)} d x+\sum_{i=1}^{n} \int_{\Omega}|u|^{\gamma(x)}\left|D_{i} u\right|^{\beta(x)} d x$, and the pseudo-norm on this space is

$$
[u]_{S_{\gamma, \beta, \theta}} \equiv \inf \left\{\lambda>0: \int_{\Omega}\left|\frac{u}{\lambda}\right|^{\theta(x)} d x+\sum_{i=1}^{n}\left(\int_{\Omega}\left|\frac{|u|^{\frac{\gamma(x)}{\beta(x)}} D_{i} u}{\lambda^{\frac{\gamma(x)}{\beta(x)}}+1}\right|^{\beta(x)}\right) d x \leq 1\right\} .
$$


Lemma 5. Assume that conditions of Proposition 2 are fulfilled. Let $u \in S_{1, \gamma(x), \beta(x), \theta(x)}(\Omega)$ and $\lambda_{u}:=[u]_{S_{\gamma, \beta, \theta}}$, then the following inequality

$$
\max \left\{\lambda_{u}^{\gamma^{-}+\beta^{-}}, \lambda_{u}^{\theta^{+}}\right\} \geq R^{\gamma, \beta, \theta}(u) \geq \min \left\{\lambda_{u}^{\gamma^{-}+\beta^{-}}, \lambda_{u}^{\theta^{+}}\right\}
$$

holds.

Theorem 2. Suppose that conditions of Proposition 2 are satisfied and let $p \in M_{0}(\Omega), p(x) \geq$ $\theta(x)$ a.e. $x \in \Omega$. Then, the embedding

$$
W^{1, p(x)}(\Omega) \subset S_{1, \gamma(x), \beta(x), \theta(x)}(\Omega)
$$

holds.

Definition 5. Let $\eta \in M_{0}(\Omega)$, we introduce $L^{1, \eta(x)}(\Omega)$ the class ${ }^{2}$ of functions $u: \Omega \rightarrow \mathbb{R}$

$$
L^{1, \eta(x)}(\Omega) \equiv\left\{u \in L^{1}(\Omega): D_{i} u \in L^{\eta(x)}(\Omega), i=\overline{1, n}\right\} .
$$

Theorem 3. Let $\gamma, \beta \in M_{0}(\Omega) \cap C^{1}(\bar{\Omega})$ and $L^{1, \beta(x)}(\Omega)$ be the space given in Definition 5 . Then the function $\varphi: \Omega \times \mathbb{R} \longrightarrow \mathbb{R}, \varphi(x, t):=|t|^{\frac{\gamma(x)}{\beta(x)}} t$ is a bijective mapping between $S_{1, \gamma(x), \beta(x), \theta(x)}(\Omega)$ and $L^{1, \beta(x)}(\Omega) \cap L^{\psi(x)}(\Omega)$, where $\psi(x):=\frac{\theta(x) \beta(x)}{\gamma(x)+\beta(x)}$.

Theorem 4. Suppose that conditions of Theorem 3 are satisfied. Let $p \in M_{0}(\Omega)$, additionally $1 \leq \beta^{-} \leq \beta(x)<n, x \in \Omega$ holds and for $\varepsilon>0$, the inequality

$$
p(x)+\varepsilon<\frac{n(\gamma(x)+\beta(x))}{n-\beta(x)}, x \in \Omega,
$$

is satisfied. Then the following compact embedding

$$
S_{1, \gamma(x), \beta(x), \theta(x)}(\Omega) \hookrightarrow L^{p(x)}(\Omega)
$$

exists.

\section{SOME RELATIONS BETWEEN CONSTANT EXPONENT PN-SPACES AND SOBOLEV SPACES}

In this section, we give some embedding results for constant exponent pn-spaces with proofs.

Theorem 5. Let $\alpha \geq 0, \beta \geq 1$. Then for all $p$ satisfying the following conditions

(i) if $\beta=n$, then $p>\beta$,

(ii) if $\beta>n$, then $p \geq \beta$,

(iii) if $\beta<n$, then $p \geq \frac{n(\alpha+\beta)}{\alpha+n}$,

the embedding

$$
W_{0}^{1, p}(\Omega) \subset \stackrel{\circ}{S}_{1, \alpha, \beta}(\Omega)
$$

holds.

\footnotetext{
${ }^{2}$ This space is not Banach one unlike to the space $W^{1, \eta(x)}(\Omega)[6]$.
} 
Proof. The cases (i) and (ii) are evident as by virtue of the Sobolev imbedding theorems occurs the inclusion

$$
W_{0}^{1, p}(\Omega) \subset C(\bar{\Omega}) .
$$

For the last case (iii), if $\beta<n$ and $p>n$, then the proof is same with the proofs of the cases (i) and (ii).

On the other side, let $\beta<n$ and $p \in\left[\frac{n(\alpha+\beta)}{\alpha+n}, n\right)$, by Sobolev imbedding theorems we have

$$
W_{0}^{1, p}(\Omega) \subset L^{\tilde{q}}(\Omega)
$$

for all $\tilde{q} \in\left[1, \frac{n p}{n-p}\right]$. Hence, for $u \in W_{0}^{1, p}(\Omega)$ we have the following estimate by Young's inequality

$$
\int_{\Omega}|u|^{\alpha}\left|D_{i} u\right|^{\beta} d x \leq\left(\frac{p-\beta}{p}\right) \int_{\Omega}|u|^{\frac{\alpha p}{p-\beta}} d x+\left(\frac{p}{\beta}\right) \int_{\Omega}\left|D_{i} u\right|^{p} d x .
$$

We deduce from the equation $\frac{\alpha p}{p-\beta}-\frac{n p}{n-p}=\frac{p[n(\alpha+\beta)-p(\alpha+n)]}{(p-\beta)(n-p)}$ and $p \in\left[\frac{n(\alpha+\beta)}{\alpha+n}, n\right)$ that

$$
\frac{\alpha p}{p-\beta} \leq \frac{n p}{n-p}
$$

Thus, by (3) and (4) we arrive at

$$
[u]_{S_{1, \alpha, \beta}^{\circ}}^{\alpha+\beta}=\int_{\Omega}|u|^{\alpha}\left|D_{i} u\right|^{\beta} d x \leq \tilde{C}\|u\|_{W_{0}^{1, p}(\Omega)}^{\frac{\alpha p}{p-\beta}}+\tilde{C}_{1}\|u\|_{W_{0}^{1, p}(\Omega)}^{p},
$$

which implies $[u]_{S_{1, \alpha, \beta}^{+}}^{\alpha+\beta} \leq \tilde{C}_{2}\|u\|_{W_{0}^{1, p}(\Omega)}^{p}+C_{3}$.

To complete the proof if $p=n>\beta$, by employing the embedding $W_{0}^{1, p}(\Omega) \subset L^{r}(\Omega)$, $1 \leq r<\infty$, one can obtain the desired result by the help of above approach.

Remark 1. Under the conditions of Theorem 5, if $p \geq \alpha+\beta$ is satisfied, then we have the imbedding (2) independently from dimension of $\Omega$.

Actually for $u \in W_{0}^{1, p}(\Omega)$, we deduce from Lemma 2 that

$$
\int_{\Omega}|u|^{\alpha}\left|D_{i} u\right|^{\beta} d x \leq C \int_{\Omega}\left|D_{i} u\right|^{p} d x+C_{1},
$$

which yields $[u]_{S_{1, \alpha, \beta}}^{\alpha+\beta} \leq C\|u\|_{W_{0}^{1, p}(\Omega)}^{p}+C_{1}$.

Theorem 6. Suppose that $\beta>\alpha \geq 0, \beta \geq 2$. Then for all $p$ satisfying the following conditions

(i) if $\alpha+\beta=n$, then $1 \leq p<2 \beta$,

(ii) if $\alpha+\beta>n$, then $1 \leq p \leq 2 \beta$,

(iii) if $\alpha+\beta<n$, then $1 \leq p \leq \frac{2 n \beta(\alpha+\beta)}{2 n \beta-(\alpha+\beta)(\beta-\alpha)}$,

the embedding

$$
\stackrel{\circ}{S}_{2, \alpha, \beta}(\Omega) \subset W_{0}^{1, p}(\Omega)
$$

holds. 
Proof. Considering these conditions, by Lemma 3 when $1 \leq p \leq \alpha+\beta$ following inequality

$$
\int_{\Omega}\left|D_{i} u\right|^{p} d x \leq C \int_{\Omega}|u|^{\alpha}\left|D_{i}^{2} u\right|^{\beta} d x+C_{1}
$$

holds independently of the dimension $n$, that yields the imbedding (5). So, if $1 \leq p \leq 2$, then $1 \leq p \leq \alpha+\beta$, which concludes the proof.

First, we prove (5) in line with conditions of (i). Let $\alpha+\beta=n$ and $p>2$ (from now on we assume $p>2$ ).

For $u \in \stackrel{\circ}{S}_{2, \alpha, \beta}(\Omega)$, by Lemma 3 we have the following estimate

$$
\int_{\Omega}\left|D_{i} u\right|^{\alpha+\beta} d x \leq C \int_{\Omega}|u|^{\alpha}\left|D_{i}^{2} u\right|^{\beta} d x .
$$

On the other hand, from Sobolev imbedding theorems

$$
W_{0}^{1, \alpha+\beta}(\Omega) \subset L^{q}(\Omega) \forall q, q \in[1, \infty) .
$$

Hence, from (6) and (7) for all $q$ satisfying $1 \leq q<\infty$ we get

$$
\|u\|_{q} \leq \tilde{C}\left(\sum_{i=1}^{n}\left\|D_{i} u\right\|_{\alpha+\beta}^{\alpha+\beta}\right)^{\frac{1}{\alpha+\beta}} \leq \tilde{C}_{0}\left(\sum_{i=1}^{n}\left[\int_{\Omega}|u|^{\alpha}\left|D_{i}^{2} u\right|^{\beta} d x\right]\right)^{\frac{1}{\alpha+\beta}}=\tilde{C}_{0}[u]_{\dot{S}_{2, \alpha, \beta}} .
$$

Therefore, for all $u \in \stackrel{\circ}{S}_{2, \alpha, \beta}(\Omega)$ and $i=\overline{1, n}$

$$
\begin{aligned}
\int_{\Omega}\left|D_{i} u\right|^{p} d x=\int_{\Omega}\left(D_{i} u\left|D_{i} u\right|^{p-2}\right) D_{i} u d x & =(p-1) \int_{\Omega} u D_{i}^{2} u\left|D_{i} u\right|^{p-2} d x \\
& \leq(p-1) \int_{\Omega}|u|^{\frac{\beta-\alpha}{\beta}}|u|^{\frac{\alpha}{\beta}}\left|D_{i}^{2} u\right|\left|D_{i} u\right|^{p-2} d x .
\end{aligned}
$$

Employing Hölder's inequality in (9) with exponents $\left(\frac{p \beta}{2 \beta-p}, \beta, \frac{p}{p-2}\right)$, we obtain

$$
\begin{aligned}
\int_{\Omega}\left|D_{i} u\right|^{p} d x & \leq C\left(\int_{\Omega}|u|^{\frac{p(\beta-\alpha)}{2 \beta-p}} d x\right)^{\frac{2 \beta-p}{p \beta}}\left(\int_{\Omega}|u|^{\alpha}\left|D_{i}^{2} u\right|^{\beta} d x\right)^{\frac{1}{\beta}}\left(\int_{\Omega}\left|D_{i} u\right|^{p} d x\right)^{\frac{p-2}{p}} \\
& =C\|u\|_{\frac{\beta(\beta-\alpha)}{2 \beta-p}}^{\frac{\beta-\alpha}{\beta}}[u]_{\dot{S}_{2, \alpha, \beta}}^{\frac{\alpha+\beta}{\beta}}\left\|D_{i} u\right\|_{p}^{p-2} .
\end{aligned}
$$

Estimating (10) by using (8) we get

$$
\int_{\Omega}\left|D_{i} u\right|^{p} d x \leq \tilde{C}[u]_{\dot{S}_{2, \alpha, \beta}}^{\frac{\beta-\alpha}{\beta}}[u]_{\dot{S}_{2, \alpha, \beta}}^{\frac{\alpha+\beta}{\beta}}\left\|D_{i} u\right\|_{p}^{p-2}=\tilde{C}[u]_{\dot{S}_{2, \alpha, \beta}}^{2}\left\|D_{i} u\right\|_{p}^{p-2} .
$$

By using Young's inequality in (11), we arrive at

$$
\left\|D_{i} u\right\|_{p}^{p} \leq \tilde{C}(\varepsilon)[u]_{S_{2, \alpha, \beta}}^{p}+\tilde{C} \varepsilon\left\|D_{i} u\right\|_{p}^{p},
$$


choosing $\varepsilon$ such that $\tilde{C} \varepsilon<1$, then we acquire

$$
\left\|D_{i} u\right\|_{p} \leq \tilde{C}[u]_{\dot{S}_{2, \alpha, \beta}}<\infty,
$$

which completes the proof for the case (i).

Assume that (ii) holds, i.e. $\alpha+\beta>n$ and $2<p \leq 2 \beta$. Then

$$
W^{1, \alpha+\beta}(\Omega) \subset C(\bar{\Omega}),
$$

by (6) and (8), we obtain

$$
\|u\|_{C(\bar{\Omega})} \leq \tilde{C}[u]_{\stackrel{S}{2, \alpha, \beta}} .
$$

For all $u \in \stackrel{\circ}{S}_{2, \alpha, \beta}(\Omega)$ from (9) one concludes

$$
\begin{aligned}
\left\|D_{i} u\right\|_{p}^{p} & \leq(p-1) \int_{\Omega}|u|^{\frac{\beta-\alpha}{\beta}}|u|^{\frac{\alpha}{\beta}}\left|D_{i}^{2} u\right|\left|D_{i} u\right|^{p-2} d x \\
& \leq(p-1) C(\varepsilon) \int_{\Omega}|u|^{\beta-\alpha}|u|^{\alpha}\left|D_{i}^{2} u\right|^{\beta} d x+(p-1) \varepsilon \int_{\Omega}\left|D_{i} u\right|^{\frac{\beta(p-2)}{\beta-1}} d x \\
& \leq(p-1) C(\varepsilon)\|u\|_{C(\bar{\Omega})}^{\beta-\alpha} \int_{\Omega}|u|^{\alpha}\left|D_{i}^{2} u\right|^{\beta} d x+(p-1) \varepsilon\left\|D_{i} u\right\|_{\frac{\beta(p-2)}{\beta-1}}^{\frac{\beta(p-2)}{\beta-1}} .
\end{aligned}
$$

By using (12) and $\frac{\beta(p-2)}{\beta-1}-p=\frac{p-2 \beta}{\beta-1}$ with $p \leq 2 \beta$ to estimate $\|u\|_{C(\bar{\Omega})}^{\beta-\alpha}$ and $\left\|D_{i} u\right\|_{\frac{\beta(p-2)}{\beta-1}}^{\frac{\beta(p-2)}{\beta-1}}$ respectively, we arrive at

$$
\begin{aligned}
\left\|D_{i} u\right\|_{p}^{p} & \leq C(\varepsilon)(p-1)[u]_{\dot{S}_{2, \alpha, \beta}^{\circ}}^{\beta-\alpha}[u]_{\dot{S}_{2, \alpha, \beta}^{\circ}}^{\alpha+\beta}+(p-1) \varepsilon \tilde{C} C\left\|D_{i} u\right\|_{p}^{p}+(p-1) \varepsilon C_{1} \\
& =C(\varepsilon)[u]_{\dot{S}_{2, \alpha, \beta}}^{2 \beta}+\varepsilon \tilde{C} C\left\|D_{i} u\right\|_{p}^{p}+\varepsilon C_{1},
\end{aligned}
$$

which implies

$$
\left\|D_{i} u\right\|_{p}^{p} \leq \tilde{C}[u]_{\dot{S}_{2, \alpha, \beta}}^{2 \beta}+C_{1}
$$

that ends the proof.

For the last case (iii), let $\alpha+\beta<n$ and $1 \leq p \leq \frac{2 n \beta(\alpha+\beta)}{2 n \beta-(\alpha+\beta)(\beta-\alpha)}$. From Sobolev imbedding theorems

$$
W^{1, \alpha+\beta}(\Omega) \subset L^{\tilde{q}}(\Omega) \quad \forall \tilde{q}, \tilde{q} \in\left[1, \frac{n(\alpha+\beta)}{n-(\alpha+\beta)}\right] .
$$

By (6) and (13), we attain

$$
\|u\|_{\tilde{q}} \leq C[u]_{\dot{S}_{2, \alpha, \beta}} .
$$

For all $u \in \stackrel{\circ}{2}_{2, \alpha, \beta}(\Omega)$, we deduce from the inequality $p \leq \frac{2 n \beta(\alpha+\beta)}{2 n \beta-(\alpha+\beta)(\beta-\alpha)}<2 \beta$ that

$$
\left\|D_{i} u\right\|_{p}^{p} \leq C\|u\|_{\frac{p(\beta-\alpha)}{2 \beta-p}}^{\frac{\beta-\alpha}{\beta}}[u]_{S_{2, \alpha, \beta}}^{\frac{\alpha+\beta}{\beta}}\left\|D_{i} u\right\|_{p}^{p-2} .
$$

If we take the inequality $\frac{p(\beta-\alpha)}{2 \beta-p} \leq \frac{n(\alpha+\beta)}{n-(\alpha+\beta)}$ into account and estimate $\|u\|_{\frac{p(\beta-\alpha)}{2 \beta-p}}$ in (15) by (14) we obtain

$$
\left\|D_{i} u\right\|_{p}^{p} \leq \tilde{C}[u]_{\dot{S}_{2, \alpha, \beta}}^{\frac{\beta-\alpha}{\beta}}[u]_{\dot{S}_{2, \alpha, \beta}}^{\frac{\alpha+\beta}{\beta}}\left\|D_{i} u\right\|_{p}^{p-2}=\tilde{C}[u]_{\dot{S}_{2, \alpha, \beta}}^{2}\left\|D_{i} u\right\|_{p}^{p-2} .
$$


Applying Young's inequality in (16) we attain

$$
\left\|D_{i} u\right\|_{p}^{p} \leq \tilde{C}(\varepsilon)[u]_{\stackrel{S}{2, \alpha, \beta}^{p}}^{p}+\tilde{C} \varepsilon\left\|D_{i} u\right\|_{p}^{p}
$$

that yields

$$
\left\|D_{i} u\right\|_{p} \leq \tilde{C}[u]_{\dot{S}_{2, \alpha, \beta}},
$$

so, the proof is complete.

We now turn our attention to some examples and results for one dimensional case.

Definition 6. Let $\alpha>\beta-1 \geq 0$ we define the following function space

$$
\begin{aligned}
\tilde{S}_{2, \alpha, \beta}(a, b):=\left\{u \in L^{1}(a, b):[u]_{\tilde{S}_{1, \alpha, \beta}(a, b)}^{\alpha+\beta}=\int_{a}^{b}|u|^{\alpha+\beta} d x\right. & +\int_{a}^{b}|u|^{\alpha-\beta}|D u|^{2 \beta} d x \\
& \left.+\int_{a}^{b}|u|^{\alpha}\left|D^{2} u\right|^{\beta} d x<\infty\right\} .
\end{aligned}
$$

The proofs of the following lemmas can be attained readily, thus we skip the proofs for the sake of brevity.

Lemma 6. Let $\tilde{S}_{2, \alpha, \beta}(a, b)$ be the space given in Definition 6 , then the imbedding

$$
\tilde{S}_{2, \alpha, \beta}(a, b) \subset S_{1, \alpha, \beta}(a, b)
$$

holds.

Lemma 7. Let $\alpha>\beta-1>0$ and $g(t) \equiv|t|^{\frac{\alpha}{\beta}} t$ for any $t \in \mathbb{R}$. Then following assertions are true

1) if $u \in \tilde{S}_{2, \alpha, \beta}(a, b)$, then $g(u) \in W^{2, \beta}(a, b)$;

2) for a function $u \in L^{1}(a, b)$, if $g(u) \equiv v \in W^{2, \beta}(a, b)$, then $u \in \tilde{S}_{2, \alpha, \beta}(a, b)$.

Consequently, we can define the space $\tilde{S}_{2, \alpha, \beta}(a, b)$ in the following way by virtue of the general definition of the nonlinear spaces.

Definition 7. Let $g: \mathbb{R} \rightarrow \mathbb{R}, g(t)=|t|^{\frac{\alpha}{\beta}} t$ and $\alpha>\beta-1>0$, then $\tilde{S}_{2, \alpha, \beta}(a, b)$ has the following representation

$$
\tilde{S}_{2, \alpha, \beta}(a, b)=\left\{u \in L^{1}(a, b):[u]_{S_{g W^{2, \beta}}^{\alpha+\beta}}^{\alpha} \equiv \sum_{0 \leq s \leq 2}\left\|D^{s} g(u)\right\|_{\beta}^{\beta}<\infty\right\} \equiv S_{g W^{2, \beta}}(a, b) .
$$

Remark 2. The following equivalences are true

$$
\tilde{S}_{2, \alpha, \beta}(a, b) \cap\left\{u:\left.u\right|_{\partial \Omega}=0\right\} \equiv \stackrel{\circ}{S}_{2, \alpha, \beta}(a, b)
$$

and

for $k=0,1$, but for $k=2$

$$
\sum_{0 \leq s \leq k}\left\|D^{s} g(u)\right\|_{\beta}^{\beta} \equiv \sum_{0 \leq s \leq k}\left\|g^{-1}\left(D^{s} g(u)\right)\right\|_{\alpha+\beta}^{\alpha+\beta}
$$

$$
\left.\left\|g^{\prime}(u) D^{2} u\right\|_{\beta}^{\beta} \equiv \| g^{-1}\left(g^{\prime}(u) D^{2} u\right)\right) \|_{\alpha+\beta}^{\alpha+\beta}
$$

and

$$
\left.\left\|g^{\prime \prime}(u)(D u)^{2}\right\|_{\beta}^{\beta} \equiv \| g^{-1}\left(g^{\prime \prime}(u)(D u)^{2}\right)\right) \|_{\alpha+\beta}^{\alpha+\beta} \text {. }
$$


The following example shows the nonlinear structure of the pn-spaces.

Example 1. Suppose that $\beta>1$. Then $S_{1,1, \beta}(0,1)$ is a nonlinear space.

Let $\tau \in\left(\frac{\beta-1}{\beta+1}, \frac{\beta-1}{\beta}\right]$ and define the functions

$$
u_{0}(x):=x^{\tau} \text { and } u_{1}(x):=\theta, x \in(0,1),\left(\theta \in \mathbb{R}^{+} \text {is a constant }\right) .
$$

It is easy to show that $u_{0}, u_{1} \in S_{1,1, \beta}(0,1)$ by the definition of $S_{1,1, \beta}(0,1)$. Besides $u(x):=u_{0}(x)+u_{1}(x)=x^{\tau}+\theta \notin S_{1,1, \beta}(0,1)$.

$$
\begin{aligned}
{[u]_{S_{1,1, \beta}(0,1)}^{\beta+1}=\int_{0}^{1}|u|^{\beta+1} d x+\int_{0}^{1}|u||D u|^{\beta} d x } & =\int_{0}^{1}\left(x^{\tau}+\theta\right)^{\beta+1} d x+\tau^{\beta} \int_{0}^{1}\left(x^{\tau}+\theta\right) x^{\beta(\tau-1)} d x \\
& =\int_{0}^{1}\left(x^{\tau}+\theta\right)^{\beta+1} d x+\tau^{\beta} \int_{0}^{1}\left(x^{\tau(\beta+1)-\beta}+\theta x^{\beta(\tau-1)}\right) d x .
\end{aligned}
$$

Since $\beta(\tau-1) \leq-1$ so, the right and side of the above equation is divergent which implies $u \notin S_{1,1, \beta}(0,1)$.

\section{VARiable EXPONENT NONLINEAR SPACES AND EMbEDDing THEOREMS}

In this section, we present certain new results with detailed proofs for variable exponent pn-spaces mentioned in Section 1. First, we derive integral inequalities (see, also [20]) to understand the structure of these spaces. Afterwards, we prove some lemmas and theorems on continuous embeddings of these spaces and on topology of them. Throughout this section, we assume that $\Omega \subset \mathbb{R}^{n}(n \geq 2)$ is a bounded domain with Lipschitz boundary.

Lemma 8. Let $\alpha, \beta \in M_{0}(\Omega)$ and $\alpha(x) \geq \beta(x)$ a.e. $x \in \Omega$. Then the inequality

$$
\int_{\Omega}|u|^{\beta(x)} d x \leq \int_{\Omega}|u|^{\alpha(x)} d x+|\Omega|, \quad \forall u \in L^{\alpha(x)}(\Omega)
$$

holds.

Proof. Let $\Omega_{1}:=\{x \in \Omega: \alpha(x)=\beta(x)\}$ and $\Omega_{2}:=\Omega \backslash \Omega_{1}$. Hence

$$
\int_{\Omega}|u|^{\beta(x)} d x=\int_{\Omega_{1}}|u|^{\alpha(x)} d x+\int_{\Omega_{2}}|u|^{\beta(x)} d x .
$$

Estimating the second integral on the right member of the above equation by utilizing Young inequality $\left(\alpha(x)>\beta(x)\right.$ on $\left.\Omega_{2}\right)$, we achieve that

$$
\int_{\Omega}|u|^{\beta(x)} d x \leq \int_{\Omega_{1}}|u|^{\alpha(x)} d x+\int_{\Omega_{2}}\left(\frac{\beta(x)}{\alpha(x)}\right)|u|^{\alpha(x)} d x+\int_{\Omega_{2}}\left(\frac{\alpha(x)-\beta(x)}{\alpha(x)}\right) d x,
$$

since $\frac{\beta(x)}{\alpha(x)}<1$ and $\frac{\alpha(x)-\beta(x)}{\alpha(x)}<1$, for $x \in \Omega_{2}$ we deduce from the last inequality that

$$
\int_{\Omega}|u|^{\beta(x)} d x \leq \int_{\Omega_{1}}|u|^{\alpha(x)} d x+\int_{\Omega_{2}}|u|^{\alpha(x)} d x+|\Omega|=\int_{\Omega}|u|^{\alpha(x)} d x+|\Omega| .
$$

On the other side if $\alpha(x)=\beta(x)$ a.e. $x \in \Omega$, then (17) is clear. 
Lemma 9. Assume that $\zeta \in M_{0}(\Omega)$ and $\beta \geq 1, \varepsilon>0$. Then for every $u \in L^{\zeta(x)+\varepsilon}(\Omega)$

$$
\int_{\Omega}|u|^{\zeta(x)}|\ln | u||^{\beta} d x \leq N_{1} \int_{\Omega}|u|^{\zeta(x)+\varepsilon} d x+N_{2}
$$

is satisfied. Here $N_{1} \equiv N_{1}(\varepsilon, \beta)>0$ and $N_{2} \equiv N_{2}(\varepsilon, \beta,|\Omega|)>0$ are constants.

Proof. Let us consider the function $f(t)=|t|^{\varepsilon}-\ln |t|$ for $t \in \mathbb{R}-\{0\}$. Since $f$ is an even function it is sufficient to investigate only $f(t)=t^{\varepsilon}-\ln t, t>0$. It can be readily shown that this function is decreasing on $\left(0, \frac{1}{\sqrt[\varepsilon]{\varepsilon}}\right]$ and increasing on the interval $\left[\frac{1}{\sqrt[\varepsilon]{\varepsilon}}, \infty\right)$. Also $f \nearrow \infty$ when $x \searrow 0$ and $x \nearrow \infty$ and $f\left(\frac{1}{\sqrt[\varepsilon]{\varepsilon}}\right)=\frac{1}{\varepsilon}(1+\ln \varepsilon)$. Here we have two situations: (i) if $\varepsilon \in\left(\frac{1}{e}, \infty\right)$, then $f\left(\frac{1}{\sqrt[\varepsilon]{\varepsilon}}\right)>0$; (ii) if $\varepsilon \in\left(0, \frac{1}{e}\right]$, then $f\left(\frac{1}{\sqrt[\varepsilon]{\varepsilon}}\right) \leq 0$. For the first case (i) $\forall t \in(0, \infty), f(t)>0$ or equivalently $\ln t<t^{\varepsilon}$. For the case (ii), the function $f$ has two zeros, say $m_{1}>0$ and $m_{2}>0$, and for $t \in \mathbb{R}^{+}-\left(m_{1}, m_{2}\right)$ it is obvious that $\ln t<t^{\varepsilon}$. For $t \in\left[m_{1}, m_{2}\right]$, $\exists N_{0}>1\left(N_{0} \equiv N_{0}\left(\frac{1}{\sqrt[\varepsilon]{\varepsilon}}\right)\right)$ such that $\ln t<N_{0} t^{\varepsilon}$. Hence, the inequality $\ln t \leq N_{0} t^{\varepsilon}$ will be satisfied on $(0, \infty)$. As a result, from the cases (i) and (ii) for arbitrary $\varepsilon>0$ and $t \in \mathbb{R}-\{0\}$, we have the inequality

$$
\ln |t| \leq N_{0}(\varepsilon)|t|^{\varepsilon}
$$

that implies on the set $\{x \in \Omega:|u(x)| \geq 1\}$ the inequality $\left.|u|^{\zeta(x)}|\ln | u\right|^{\beta} \leq N_{0}(\varepsilon, \beta)|u|^{\zeta(x)+\varepsilon}$ be fulfilled. Moreover, from $\lim _{t \rightarrow 0^{+}} t^{\varepsilon}|\ln t|^{\beta}=0$ and for every fixed $x_{0} \in \Omega, \lim _{t \rightarrow 0^{+}} \frac{|t|^{\zeta\left(x_{0}\right)}|\ln | t||^{\beta}}{t^{\zeta\left(x_{0}\right)+\varepsilon}+1}=0$, we arrive at the inequality $\left.|u|^{\zeta(x)-1}|u||\ln | u\right|^{\beta} \leq \tilde{N}_{0}\left(|u|^{\zeta(x)+\varepsilon}+1\right)$ is fulfilled on the set $\{x \in \Omega:|u(x)|<1\}$ for some $\tilde{N}_{0}=\tilde{N}_{0}(\varepsilon, \beta)>0$. So, the proof is complete by the combination of these inequalities.

Lemma 10. Let $\tilde{\varepsilon}>0$ and $\beta_{1}: \Omega \rightarrow[\tilde{\varepsilon}, \infty)$ be a measurable function, which satisfies $\tilde{\varepsilon} \leq \beta_{1}^{-} \leq \beta_{1}(x) \leq \beta_{1}^{+}<\infty$ and $\xi, \beta \in M_{0}(\Omega)$, then the inequality

$$
\int_{\Omega}|u|^{\xi(x)}|\ln | u||^{\beta(x)} d x \leq C_{1} \int_{\Omega}|u|^{\xi(x)+\beta_{1}(x)} d x+C_{2}, \forall u \in L^{\xi(x)+\beta_{1}(x)}(\Omega)
$$

holds. Here $C_{1} \equiv C_{1}\left(\tilde{\varepsilon}, \beta^{+}\right)>0$ and $C_{2} \equiv C_{2}\left(\tilde{\varepsilon}, \beta^{+},|\Omega|\right)>0$ are constants.

Proof. For arbitrary $\gamma \in(0,1), \frac{\beta^{+}+\gamma}{\beta(x)}>1$, by utilizing the Young's inequality with this exponent to $|\ln | u||^{\beta(x)}$ we obtain the following inequality $|\ln | u||^{\beta(x)} \leq\left.|\ln | u\right|^{\beta^{+}+\gamma}+1$, by multiplying each side of this inequality with $|u|^{\xi(x)}$, we get

$$
|u|^{\xi(x)}|\ln | u||^{\beta(x)} \leq|u|^{\xi(x)}|\ln | u||^{\beta^{+}+\gamma}+|u|^{\xi(x)}, x \in \Omega .
$$

Thus, integrating both sides over $\Omega$,

$$
\int_{\Omega}|u|^{\xi(x)}|\ln | u||^{\beta(x)} d x \leq \int_{\Omega}|u|^{\xi(x)}|\ln | u||^{\beta^{+}+\gamma} d x+\int_{\Omega}|u|^{\xi(x)} d x
$$

is established. For $\varepsilon<\tilde{\varepsilon}$, estimating the first integral on the right side of the last inequality by Lemma 9, we acquire

$$
\int_{\Omega}|u|^{\xi(x)}|\ln | u||^{\beta(x)} d x \leq C_{3} \int_{\Omega}|u|^{\xi(x)+\varepsilon} d x+C_{4}+\int_{\Omega}|u|^{\xi(x)} d x .
$$


As $\frac{\xi(x)+\varepsilon}{\xi(x)}>1$, applying Lemma 8 to estimate the second integral on the right member of the last inequality, we gain

$$
\int_{\Omega}|u|^{\xi(x)}|\ln | u||^{\beta(x)} d x \leq C_{1} \int_{\Omega}|u|^{\xi(x)+\varepsilon} d x+C_{2},
$$

here $C_{1} \equiv C_{1}\left(\varepsilon, \beta^{+}\right)>0$ and $C_{2} \equiv C_{2}\left(\varepsilon, \beta^{+},|\Omega|\right)>0$ are constants.

Since $\xi(x)+\varepsilon<\xi(x)+\beta_{1}(x)$, a.e. $x \in \Omega$, estimating the integral on the right side of the above equation by using Lemma 8 , we attain (18).

In the following discussions, we examine elaborate properties of the pn-spaces $S_{1, \gamma(x), \beta(x), \theta(x)}(\Omega)$, presented in Section 1 (for other results, see $[19,20]$ ).

Lemma 11. Let $S_{1, \gamma(x), \beta(x), \theta(x)}(\Omega)$ and $S_{1, \xi(x), \alpha(x), \theta_{1}(x)}(\Omega)$ be the spaces given in Definition 4 . Assume that one of the conditions given below are satisfied

(i) $\theta_{1}(x) \leq \theta(x), \beta(x) \geq \alpha(x)$ and $\xi(x) \beta(x)=\gamma(x) \alpha(x)$, a.e. $x \in \Omega$,

(ii) $\theta_{1}(x) \leq \theta(x), \xi(x) \beta(x)>\gamma(x) \alpha(x), \gamma(x)+\beta(x) \geq \xi(x)+\alpha(x)$ and $\beta(x) \geq \alpha(x)+$ $\varepsilon$ for some $\varepsilon>0$.

Under these conditions the embedding

$$
S_{1, \gamma(x), \beta(x), \theta(x)}(\Omega) \subset S_{1, \xi}(x), \alpha(x), \theta_{1}(x)(\Omega)
$$

holds.

Proof. First, suppose that (i) holds. Let $u \in S_{1, \gamma(x), \beta(x), \theta(x)}(\Omega)$, to show the embedding (19) it is sufficient to verify the finiteness of

$$
R^{\xi, \alpha, \theta_{1}}(u)=\int_{\Omega}|u|^{\theta_{1}(x)} d x+\sum_{i=1}^{n} \int_{\Omega}|u|^{\xi(x)}\left|D_{i} u\right|^{\alpha(x)} d x,
$$

estimating the first integral on the right member of the above equation with the help of Lemma 8 and second one by employing Young's inequality, we acquire

$$
R^{\xi, \alpha, \theta_{1}}(u) \leq(n+1)|\Omega|+\int_{\Omega}|u|^{\theta(x)} d x+\sum_{i=1}^{n} \int_{\Omega}|u|^{\frac{\xi(x) \beta(x)}{\alpha(x)}}\left|D_{i} u\right|^{\beta(x)} d x .
$$

From the conditions, $\frac{\xi(x) \beta(x)}{\alpha(x)}=\gamma(x)$ that yields

$$
R^{\xi, \alpha, \theta_{1}}(u) \leq R^{\gamma, \beta, \theta}(u)+(n+1)|\Omega|,
$$

so (19) is gained. We note that when the case $\beta(x)=\alpha(x)$ a.e. $x \in \Omega$, then $\xi(x)=\gamma(x)$, hence (19) can be obtained by similar operations as above.

Now, assume that (ii) fulfills. We need to show that $R^{\xi, \alpha, \theta_{1}}(u)$ is finite. We have

$$
\begin{aligned}
R^{\xi, \alpha, \theta_{1}}(u) & =\int_{\Omega}|u|^{\theta_{1}(x)} d x+\sum_{i=1}^{n} \int_{\Omega}|u|^{\xi(x)}\left|D_{i} u\right|^{\alpha(x)} d x \\
& =\int_{\Omega}|u|^{\theta_{1}(x)} d x+\sum_{i=1}^{n} \int_{\Omega}|u|^{\xi(x)-\frac{\gamma(x) \alpha(x)}{\beta(x)}}|u|^{\frac{\gamma(x) \alpha(x)}{\beta(x)}}\left|D_{i} u\right|^{\alpha(x)} d x .
\end{aligned}
$$


If we estimate the first integral on the right member of the above equation with the help of Lemma 8 and second one by employing Young's inequality with the exponent $\frac{\beta(x)}{\alpha(x)}$ at every point, one can acquire that

$$
R^{\xi, \alpha, \theta_{1}}(u) \leq \int_{\Omega}|u|^{\theta(x)} d x+|\Omega|+\sum_{i=1}^{n} \int_{\Omega}|u|^{\gamma(x)}\left|D_{i} u\right|^{\beta(x)} d x+n \int_{\Omega}|u|^{\frac{\xi(x) \beta(x)-\gamma(x) \alpha(x)}{\beta(x)-\alpha(x)}} d x .
$$

In the light of the condition (ii), the inequality $\frac{\xi(x) \beta(x)-\gamma(x) \alpha(x)}{\beta(x)-\alpha(x)}<\gamma(x)+\beta(x)$ holds, so estimating the third integral in the right side of the last inequality by Lemma 8 , we arrive at

$$
\begin{aligned}
R^{\xi, \alpha, \theta_{1}}(u) & \leq(n+1) \int_{\Omega}|u|^{\theta(x)} d x+(n+1)|\Omega|+\sum_{i=1}^{n} \int_{\Omega}|u|^{\gamma(x)}\left|D_{i} u\right|^{\beta(x)} d x \\
& \leq(n+1)\left(R^{\gamma, \beta, \theta}(u)+|\Omega|\right),
\end{aligned}
$$

hence from here desired inequality is achieved. Also if $\theta_{1}(x)=\theta(x)$ a.e. $x \in \Omega$, by employing the same operations one can show (19).

Lemma 12. Let $\beta, \gamma$ and $\psi$ satisfy the conditions of Theorem 3 , then $S_{1, \gamma(x), \beta(x), \theta(x)}(\Omega)$ is a metric space with the metric which is defined below

$$
d_{S_{1}}(u, v):=\|\varphi(u)-\varphi(v)\|_{L^{\psi(x)}(\Omega)}+\sum_{i=1}^{n}\left\|\varphi_{t}^{\prime}(u) D_{i} u-\varphi_{t}^{\prime}(v) D_{i} u\right\|_{L^{\beta(x)}(\Omega)},
$$

$\forall u, v \in S_{1, \gamma(x), \beta(x), \theta(x)}(\Omega)$, here $\varphi(x, t)=|t|^{\frac{\gamma(x)}{\beta(x)}} t$ and for every fixed $x \in \Omega$

$$
\varphi_{t}^{\prime}(t)=\left(\frac{\gamma(x)}{\beta(x)}+1\right)|t|^{\frac{\gamma(x)}{\beta(x)}}
$$

Proof. It has been shown in Theorem 3 that $^{3} \varphi(u) \in L^{\psi(x)}(\Omega)$ and $\varphi_{t}^{\prime}(u) D_{i} u \in L^{\beta(x)}(\Omega)$ whenever $u \in S_{1, \gamma(x), \beta(x), \theta(x)}(\Omega)$, thus one can verify that $d_{S_{1}}(\cdot, \cdot): S_{1, \gamma(x), \beta(x), \theta(x)}(\Omega) \rightarrow \mathbb{R}$ satisfy the metric axioms, i.e.

(i) $d_{S_{1}}(u, v) \geq 0$,

(ii) $d_{S_{1}}(u, v)=d_{S_{1}}(v, u)$,

(iii) $u=v \Rightarrow d_{S_{1}}(u, v)=0$,

(iv) $d_{S_{1}}(u, v)=0 \Rightarrow\|\varphi(u)-\varphi(v)\|_{L^{\psi(x)}(\Omega)}=0 \Rightarrow \varphi(u)=\varphi(v)$ since $\varphi$ is 1-1, then $u=v$,

(v) from the subadditivity of norm, $d_{S_{1}}(u, v) \leq d_{S_{1}}(u, w)+d_{S_{1}}(w, v)$.

\footnotetext{
${ }^{3}$ From now on, we denote $\varphi(x, u):=\varphi(u)=|u|^{\frac{\gamma(x)}{\beta(x)}} u$ for simplicity.
} 
Theorem 7. Under the conditions of Theorem 3, $\varphi$ is a homeomorphism between the spaces $S_{1, \gamma(x), \beta(x), \theta(x)}(\Omega)$ and $L^{1, \beta(x)}(\Omega) \cap L^{\psi(x)}(\Omega)$.

Proof. The function $\varphi$ is a bijection between $S_{1, \gamma(x), \beta(x), \theta(x)}(\Omega)$ and $L^{1, \beta(x)}(\Omega) \cap L^{\psi(x)}(\Omega)$ by Theorem 3. Thus it is ample to prove the continuity of $\varphi$ as well as $\varphi^{-1}$ in the sense of topology induced by the metric $d_{S_{1}}(\cdot, \cdot)$. For this, we need to show that

(i) $d_{S_{1}}\left(u_{m}, u_{0}\right) \underset{m{ }_{\infty}}{\longrightarrow} 0 \Rightarrow \varphi\left(u_{m}\right) \underset{L^{1, \beta(x)} \underset{(\Omega) \cap L^{\psi(x)}}{\longrightarrow}(\Omega)}{\longrightarrow} \varphi\left(u_{0}\right)$ for every $\left\{u_{m}\right\} \in S_{1, \gamma(x), \beta(x), \theta(x)}(\Omega)$ which converges to $u_{0}$ and

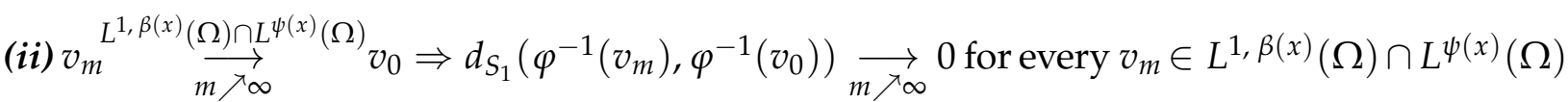
which converges to $v_{0}$.

Since for every $v_{m}$ and $v_{0}$ there exist a unique $u_{m}$ and $u_{0} \in S_{1, \gamma(x), \beta(x), \theta(x)}(\Omega)$ such that $\varphi\left(u_{m}\right)=v_{m}$ and $\varphi\left(u_{0}\right)=v_{0}$, the implication (ii) can be written equivalently as follows $\varphi\left(u_{m}\right) \underset{m \nearrow_{\infty}}{\stackrel{L^{1, \beta(x)}(\Omega) \cap L^{\psi(x)}(\Omega)}{\longrightarrow}} \varphi\left(u_{0}\right) \Rightarrow d_{S_{1}}\left(u_{m}, u_{0}\right) \underset{m{ }^{\infty}}{\longrightarrow} 0$ for every $\left\{u_{m}\right\} \in S_{1, \gamma(x), \beta(x), \theta(x)}(\Omega)$ which converges to $u_{0}$.

Since the proofs of $(i)$ and (ii) are similar, we only prove (ii). Let $v_{0}, v_{m} \in L^{1, \beta(x)}(\Omega) \cap$ $L^{\psi(x)}(\Omega)$ and $v_{m} \stackrel{L^{1, \beta(x)}(\Omega) \cap L^{\psi(x)}(\Omega)}{\longrightarrow} v_{0} \Leftrightarrow \varphi\left(u_{m}\right) \stackrel{L^{1, \beta(x)}(\Omega) \cap L^{\psi(x)}(\Omega)}{\longrightarrow} \varphi\left(u_{0}\right)$.

To verify $d_{S_{1}}\left(u_{m}, u_{0}\right) \rightarrow 0$, by definition of metric $d_{S_{1}}$ it is ample to demonstrate that

$$
\left\|\varphi_{t}^{\prime}\left(u_{m}\right) D_{i} u_{m}-\varphi_{t}^{\prime}\left(u_{0}\right) D_{i} u_{0}\right\|_{L^{\beta(x)}(\Omega)} \rightarrow 0 \quad \text { and }\left\|\varphi\left(u_{m}\right)-\varphi\left(u_{0}\right)\right\|_{L^{\psi(x)}(\Omega)} \rightarrow 0
$$

as $m \nearrow \infty$.

From $\varphi\left(u_{m}\right) \stackrel{L^{1, \beta(x)}(\Omega) \cap L^{\psi(x)}(\Omega)}{\longrightarrow} \varphi\left(u_{0}\right)$, we have

$$
\left\|\varphi\left(u_{m}\right)-\varphi\left(u_{0}\right)\right\|_{L^{\psi(x)}(\Omega)} \rightarrow 0 \quad \text { and } \quad\left\|D_{i}\left(\varphi\left(u_{m}\right)-\varphi\left(u_{0}\right)\right)\right\|_{L^{\beta(x)}(\Omega)} \rightarrow 0 .
$$

Hence, we only need to show that

$$
\left\|\varphi_{t}^{\prime}\left(u_{m}\right) D_{i} u_{m}-\varphi_{t}^{\prime}\left(u_{0}\right) D_{i} u_{0}\right\|_{L^{\beta(x)}(\Omega)} \longrightarrow 0 \text { as } m \nearrow \infty .
$$

From Lemma 4, we have

$$
\left\|\varphi_{t}^{\prime}\left(u_{m}\right) D_{i} u_{m}-\varphi_{t}^{\prime}\left(u_{0}\right) D_{i} u_{0}\right\|_{L^{\beta(x)}(\Omega)} \rightarrow 0 \Leftrightarrow \sigma_{\beta}\left(\varphi_{t}^{\prime}\left(u_{m}\right) D_{i} u_{m}-\varphi_{t}^{\prime}\left(u_{0}\right) D_{i} u_{0}\right) \rightarrow 0 .
$$

Based on (20), for $i=\overline{1, n}$

$$
\sigma_{\beta}\left(\varphi_{t}^{\prime}\left(u_{m}\right) D_{i} u_{m}-\varphi_{t}^{\prime}\left(u_{0}\right) D_{i} u_{0}\right)=\int_{\Omega}\left|\varphi_{t}^{\prime}\left(u_{m}\right) D_{i} u_{m}-\varphi_{t}^{\prime}\left(u_{0}\right) D_{i} u_{0}\right|^{\beta(x)} d x,
$$

one can show that the following equality holds

$$
\begin{aligned}
\varphi_{t}^{\prime}\left(u_{m}\right) D_{i} u_{m}-\varphi_{t}^{\prime}\left(u_{0}\right) D_{i} u_{0} & =\left(\frac{\beta(x)}{\beta(x)+\gamma(x)}\right) D_{i}\left(\varphi\left(u_{m}\right)-\varphi\left(u_{0}\right)\right) \\
& -\left(\frac{D_{i} \gamma \cdot \beta-\gamma \cdot D_{i} \beta}{\beta(\gamma+\beta)}\right)\left(\left|u_{m}\right|^{\frac{\gamma(x)}{\beta(x)}} u_{m} \ln \left|u_{m}\right|-\left|u_{0}\right|^{\frac{\gamma(x)}{\beta(x)}} u_{0} \ln \left|u_{0}\right|\right) .
\end{aligned}
$$


Substituting (22) into (21), we acquire

$$
\begin{aligned}
\sigma_{\beta}\left(\varphi_{t}^{\prime}\left(u_{m}\right) D_{i} u_{m}-\varphi_{t}^{\prime}\left(u_{0}\right) D_{i} u_{0}\right) & \\
=\int_{\Omega} & \mid\left(\frac{\beta(x)}{\gamma(x)+\beta(x)}\right) D_{i}\left(\varphi\left(u_{m}\right)-\varphi\left(u_{0}\right)\right) \\
& \quad-\left.\left(\frac{D_{i} \gamma \cdot \beta-\gamma \cdot D_{i} \beta}{\beta(\gamma+\beta)}\right)\left(\left|u_{m}\right|^{\frac{\gamma(x)}{\beta(x)}} u_{m} \ln \left|u_{m}\right|-\left|u_{0}\right|^{\frac{\gamma(x)}{\beta(x)}} u_{0} \ln \left|u_{0}\right|\right)\right|^{\beta(x)}
\end{aligned}
$$

taking $\beta(x)$ into the absolute value and applying known inequality, we gain

$$
\begin{aligned}
\sigma_{\beta}\left(\varphi_{t}^{\prime}\left(u_{m}\right) D_{i} u_{m}-\varphi_{t}^{\prime}\left(u_{0}\right) D_{i} u_{0}\right) & \leq 2^{\beta^{+}-1} \int_{\Omega}\left|D_{i}\left(\varphi\left(u_{m}\right)\right)-D_{i}\left(\varphi\left(u_{0}\right)\right)\right|^{\beta(x)} d x \\
& +C_{3} \int_{\Omega}\left|u_{m}\right|^{\frac{\gamma(x)}{\beta(x)}} u_{m} \ln \left|u_{m}\right|-\left.\left|u_{0}\right|^{\frac{\gamma(x)}{\beta(x)}} u_{0} \ln \left|u_{0}\right|\right|^{\beta(x)} d x,
\end{aligned}
$$

here $C_{3}=C_{3}\left(\beta^{+},\|\gamma\|_{C^{1}(\bar{\Omega})},\|\beta\|_{C^{1}(\bar{\Omega})}\right)>0$ is constant.

Since $\left\|D_{i}\left(\varphi\left(u_{m}\right)-\varphi\left(u_{0}\right)\right)\right\|_{L^{\beta(x)}(\Omega)} \longrightarrow 0$ as $m \nearrow \infty$, the first integral in the right member of (23) converges to zero when $m$ tends to infinity (Lemma 4 ).

From Theorem 3, function $\varphi$ is bijective between the spaces $L^{\theta(x)}(\Omega)$ and $L^{\psi(x)}(\Omega)$. Also since $\left\|\varphi\left(u_{m}\right)-\varphi\left(u_{0}\right)\right\|_{L^{\psi(x)}(\Omega)} \longrightarrow 0$, we arrive at

$$
\varphi\left(u_{m}\right) \underset{\Omega}{\stackrel{a . e}{\longrightarrow}} \varphi\left(u_{0}\right) \Rightarrow u_{m} \underset{\Omega}{\stackrel{a . e}{\longrightarrow}} u_{0}
$$

and

$$
\sigma_{\theta}\left(u_{m}\right)=\int_{\Omega}\left|u_{m}\right|^{\theta(x)} d x=\left.\left.\int_{\Omega}|| u_{m}\right|^{\frac{\gamma(x)}{\beta(x)}} u_{m}\right|^{\psi(x)} d x=\int_{\Omega}\left|\varphi\left(u_{m}\right)\right|^{\psi(x)} d x \leq M
$$

for some $M>0$.

Employing (24), (25) and Vitali's Theorem ${ }^{4}$, we attain

$$
\int_{\Omega}\left|u_{m}\right|^{\theta(x)} d x \longrightarrow \int_{\Omega}\left|u_{0}\right|^{\theta(x)} d x, \quad m \nearrow \infty
$$

Since $u_{m}$ converges to $u_{0}$ in measure on $\Omega$, using this and (26), we deduce from Lemma 4 that

$$
\sigma_{\theta}\left(u_{m}-u_{0}\right) \longrightarrow 0 \Rightarrow\left\|u_{m}-u_{0}\right\|_{L^{\theta(x)}(\Omega)} \longrightarrow 0 .
$$

\footnotetext{
${ }^{4}$ Theorem (Vitali, [16]). Let $(\Omega, \Sigma, \mu)$ be a finite measure space, and $f_{n}: \Omega \rightarrow \mathbb{R}$ be a sequence of measurable functions converging a.e. to a measurable $f$. Then $\left\|f_{n}-f\right\|_{L^{1}(\Omega)} \rightarrow 0$ as $n \rightarrow \infty$ iff $\left\{f_{n}: n \geq 1\right\}$ is uniformly integrable. When the condition is satisfied, we have

$$
\lim _{n \rightarrow \infty} \int_{\Omega} f_{n} d \mu=\int_{\Omega} f d \mu
$$
}


Denote $w_{m}:=\left|u_{m}\right|^{\frac{\gamma(x)}{\beta(x)}} u_{m} \ln \left|u_{m}\right|$ and $w_{0}:=\left|u_{0}\right|^{\frac{\gamma(x)}{\beta(x)}} u_{0} \ln \left|u_{0}\right|$, then

$$
\sigma_{\beta}\left(w_{m}\right)=\int_{\Omega}\left|u_{m}\right|^{\gamma(x)+\beta(x)}|\ln | u_{m}||^{\beta(x)} d x .
$$

Estimating the above integral by using Lemma 10, one can obtain

$$
\sigma_{\beta}\left(w_{m}\right) \leq C_{4} \int_{\Omega}\left|u_{m}\right|^{\theta(x)} d x+C_{5}=C_{4} \sigma_{\theta}\left(u_{m}\right)+C_{5} .
$$

From (27), $\sigma_{\beta}\left(w_{m}\right) \leq \tilde{M}$ for all $m \geq 1$, for some $\tilde{M}>0$. Thus as shown above for $u_{m}$ similarly we conclude that as $m \nearrow \infty$

$$
\left.\sigma_{\beta}\left(w_{m}-w_{0}\right) \longrightarrow 0 \Rightarrow \int_{\Omega}|| u_{m}\right|^{\frac{\gamma(x)}{\beta(x)}} u_{m} \ln \left|u_{m}\right|-\left.\left|u_{0}\right|^{\frac{\gamma(x)}{\beta(x)}} u_{0} \ln \left|u_{0}\right|\right|^{\beta(x)} \longrightarrow 0,
$$

hence from (23) we attain,

$$
\left\|\varphi_{t}^{\prime}\left(u_{m}\right) D_{i} u_{m}-\varphi_{t}^{\prime}\left(u_{0}\right) D_{i} u_{0}\right\|_{L^{\beta(x)}(\Omega)} \longrightarrow 0, \quad m \nearrow \infty .
$$

So, the proof is complete.

\section{REFERENCES}

[1] Adams R.A. Sobolev Spaces. Academic Press, New York, 1975.

[2] Antontsev S.N., Rodrigues J.F. On stationary thermo-rheological viscous flows. Ann. Univ. Ferrara Sez. VII Sci. Mat. 2006, 52 (1), 19-36. doi:10.1007/s11565-006-0002-9

[3] Berestycki H., Nirenberg L. Some qualitive properties of solutions of semilinear elliptic equations in cylindrical domains. Analysis, Academic Press Boston, 1990.

[4] Brezis H. Points critiques dans les problèmes variationnels sans compacité. Séminaire Bourbaki 1989, 698 (5), 239256.

[5] Chen Y., Levine S., Rao M. Variable exponent, linear growth functionals in image restoration. SIAM J. Appl. Math. 2006, 66 (4), 1383-1406. doi:10.1137/050624522

[6] Diening L., Harjulehto P., Hastö P., Ružička M. Lebesgue and Sobolev Spaces with Variable Exponents. Lecture Notes in Mathematics, Springer, Heidelberg 2011. doi:10.1007/978-3-642-18363-8

[7] Dubinskiǔ Yu.A. Weak convergence in nonlinear elliptic and parabolic equations. Mat. Sb., 1965, 67 (109), 609-642.

[8] Dubinskir Yu.A. Nonlinear elliptic and parabolic equations. J. Math. Sci. 1979, 12, 475-554. doi: 10.1007/BF01089137 (translation of Itogi Nauki i Tekhniki. Ser. Sovrem. Probl. Mat. 1976, 9, 5-130. (in Russian))

[9] Fan X., Zhao D. On the spaces $L^{p(x)}(\Omega)$ and $W^{k, p(x)}(\Omega)$. J. Math. Anal. Appl. 2001, 263 (2), 424-446. doi:10.1006/jmaa.2000.7617

[10] Kováčik O., Rákosník J. On spaces $L^{p(x)}$ and $W^{k, p(x)}$. Czechoslovak Math. J. 1991, 41 (4), 592-618.

[11] Lions J.L. Quelques méthodes de résolution des problèmes aux limites non linéaires. Dunod and GauthierVillars, Paris, 1969. 
[12] Luckhaus S., Passo R. Dal. A degenerate diffusion problem not in divergence form. J. Differential Equations 1987, 69 (1), 1-14.

[13] Musielak J. Orlicz Spaces and Modular Spaces. Lecture Notes in Mathematics, Springer-Verlag, Berlin, 1983. doi:10.1007/BFb0072210

[14] Oleinnik O.A. The mathematical problems of the theory of the boundary layer. Russ. Math. Surv. 1968, 23 (3), 3-65. (in Russian)

[15] Pohozaev S.I. Nonlinear operators which have a weakly closed range of values and quasilinear elliptic equations. Mat. Sb. 1969, 78, 237-259.

[16] Rao M.M. Measure Theory and Integration. John Wiley \& Sons, New York, 1984.

[17] Ruzicka M. Electrorheological Fluids: Modeling and Mathematical Theory. In: Lecture Notes in Mathematics, Springer-Verlag, Berlin, 2000. doi:10.1007/BFb0104029

[18] Samokhin B.N. The system of equations of a boundary layer of a pseudoplastic fluid. Dokl. Akad. Nauk SSSR 1973, 210 (5), 1043-1046. (in Russian)

[19] Sert U., Soltanov K.N. On Solvability of a Class of Nonlinear Elliptic type Equation with Variable Exponent. J. Appl. Anal. Comput. 2017, 7 (3), 1139-1160. doi:10.11948/2017071

[20] Sert U., Soltanov K.N. Solvability of nonlinear elliptic type equation with two unrelated non standard growths. J. Korean Math. Soc. 2018, 55 (6), 1337-1358. doi:10.4134/JKMS.j170691

[21] Soltanov K.N. Imbedding theorems of the nonlinear spaces and solvability some nonlinear noncoercive equations. Preprint, VINITI, N3696-B 91, Moscow, 1991, 71 pp. (in Russian)

[22] Soltanov K.N. Some embedding theorems and its applications to nonlinear equations. Differ. Uravn. 1984, 20 (12), 2181-2184. (in Russian)

[23] Soltanov K.N. Some applications of the nonlinear analysis to the differential equations. ELM, Baku, 2002. (in Russian)

[24] Soltanov K.N. Periodic solutions some nonlinear parabolic equations with implicit degenerate. Dokl. Akad. Nauk SSSR 1975, 222 (2), 291-294. (in Russian)

[25] Soltanov K.N. Embedding theorems for nonlinear spaces and solvability of some nonlinear noncoercive equations. Proc. Inst. Math. Mech. Natl. Acad. Sci. Azerb. 1996, 5, 72-103. (in Russian)

[26] Soltanov K.N. Some nonlinear equations of the nonstable filtration type and embedding theorems. Nonlinear Anal. 2006, 65 (11), 2103-2134. doi:10.1016/j.na.2005.11.053

[27] Soltanov K.N. Existence and nonexistence of the global solutions some nonlinear elliptic-parabolic equations. Differ. Uravn. 1993, 29 (4), 646-661. (in Russian)

[28] Soltanov K.N. On nonlinear equations of the form $F(x, u, D u, \Delta u)=0$. Sb. Math. 1995, 80 (2), 367-392.

[29] Soltanov K.N. Solvability nonlinear equations with operators the form of sum the pseudomonotone and weakly compact. Dokl. Akad. Nauk 1992, 324 (5), 944-948.

[30] Soltanov K.N. Solvability of certain parabolic problems with faster-growing-than-power nonlinearities. Mat. Zametki 1982, 32 (6), 909-923.

[31] Soltanov K.N. Smooth solvability some quasielliptic problems. Izv. Akad. Nauk Azerbaĭdzhan. 1979, 2, 61-67. (in Russian)

[32] Soltanov K.N. Some Boundary Problem for Emden-Fowler Type Equations, Function Spaces. Differential Operators and Nonlinear Analysis, "FSDONA", Praha, Czech Republic, May, 2005, Math. Inst. Acad. Sci., Praha, Czech Republic, 2005, 311-318.

[33] Soltanov K.N., Akmedov M. Solvability of Equation of Prandtl-von Mises type, Theorems of Embedding. Trans. Natl. Acad. Sci. Azerb. Ser. Phys.-Tech. Math. Sci. 2017, 37 (1), 143-168.

[34] Soltanov K.N., Sprekels J. Nonlinear equations in nonreflexive Banach spaces and fully nonlinear equations. Adv. Math. Sci. Appl. 1999, 9 (2), 939-972. 
[35] Tsutsumi T., Ishiwata M. Regional blow-up of solutions to initial boundary value problem for $u_{t}=u^{\delta}(\Delta u+u)$. Proc. Roy. Soc. Edinburgh Sect. A 1997, 127 (4), 871-887.

[36] Walter W. Existence and convergence theorems for the boundary layer equations based on the line method. Arch. Ration. Mech. Anal. 1970, 39 (3), 169-188.

[37] Wiegner M. A degenerate diffusion equation with a nonlinear source term. Nonlinear Anal. 1997, 28 (12), 19771995. doi:10.1016/S0362-546X(96)00027-2

[38] Zhikov V.V. On some variational problems. Russ. J. Math. Phys. 1997, 5 (1), 105-116.

Received 16.07.2019

Солтанов К., Серт У. Аеякі результати для одного класу нелінійних функціональних просторів // Карпатські матем. публ. — 2020. - Т.12, №1. — С. 208-228.

У даній роботі ми вивчаємо властивості класу функціональних просторів, так званих pn-просторів, які з'являються при дослідженні нелінійних диференщіальних рівнянь. Ми встановили деякі інтегральні нерівності для аналізу структури рп-просторів зі сталими та змінними показниками. Ми довели теореми про вкладення, які встановлюють співвідношення цих просторів з добре відомими класичними просторами Аебега і Соболєва зі сталими та змінними показниками.

Ключові слова і фрази: рn-простір, змінний показник, інтегральна нерівність, нелінійне диференціальне рівняння, теорема про вклалення. 